\title{
Immunohistochemical study of the distribution of adrenergic and peptidergic innervation in the equine uterus and the cervix
}

\author{
S-E. Bae, B. M. Corcoran and E. D. Watson \\ Department of Veterinary Clinical Studies, Royal (Dick) School of Veterinary Studies, \\ University of Edinburgh, Easter Bush Veterinary Center, Roslin, Midlothian, EH25 9RG, UK
}

Little is known about neurogenic regulation of uterine contractility in mares. The present study investigated the distribution of adrenergic and peptidergic nerves in the mare uterus. Samples from the uterine horn, body and cervix were collected from 18 cyclic mares for immunohistochemistry. The uterus was well supplied with adrenergic nerves. A large number of tyrosine hydroxylase- and dopamine $\beta$-hydroxylase-immunoreactive nerve bundles and fibres were present in the myometrium and endometrium in all regions of the uterus and cervix. These adrenergic nerve bundles and fibres travelled parallel to the muscle layers and were often associated with blood vessels. The density of peptidergic nerves was less than that of adrenergic nerves, but the pattern of distribution was similar. Neuropeptide $\mathrm{Y}$ immunoreactive nerve fibres were the most abundant, whereas vasoactive intestinal polypeptide- and calcitonin gene-related peptide-immunoreactive nerve fibres were less frequently seen. Substance P-immunoreactive nerve fibres were the most sparse. Peptidergic nerves were distributed among the smooth muscle layers and near endometrial glands and were often associated with blood vessels in all regions of the uterus. The density of peptidergic nerve fibres was similar in the uterine horn and body but was slightly denser in the cervix. These findings indicate that uterine innervation may have an important role in controlling reproductive functions in mares.

\section{Introduction}

Uterine contractility is regulated by the coordination and interaction of myogenic, neurogenic and hormonal control mechanisms. The main external factors that control uterine contractility are the sex steroid hormones, oestrogen and progesterone, and also oxytocin and prostaglandins. In mares, uterine contractility is of key importance in uterine clearance after mating (Troedsson et al., 1993). Mares susceptible to endometritis induced by persistent mating may have impaired uterine contractile activity (Troedsson et al., 1993; Nikolakopoulos and Watson, 1997) and defects in innervation or nerve function may contribute to this condition. However, there have been no detailed studies on innervation of the equine uterus.

Previous studies on innervation of the mammalian female reproductive tract have demonstrated that it is well supplied by the autonomic nervous system, with adrenergic nerve fibres predominating (Thorbert et al., 1977; Alm and Lundberg, 1988; Mitchell and Ahmed, 1992). Tyrosine hydroxylase $(\mathrm{TH})$ and dopamine $\beta$-hydroxylase $(\mathrm{D} \beta \mathrm{H})$ are catecholamine-synthesizing enzymes and their presence has been used to demonstrate the adrenergic contribution to uterine innervation (Alm and Lundberg, 1988; Wrobel and Kujat, 1993). In contrast to other organs, uterine adrenergic innervation is affected by ovarian steroid hormones during

Email:dvmsbae@hotmail.com the oestrous cycle and pregnancy (Marshall, 1981; Zoubina et al., 1998). Adrenergic innervation is important in the neuronal control of uterine blood flow, myometrial contractility, and endometrial secretory function (Heinrich et al., 1986) and exerts biological effects consistent with a regulatory function in female reproduction (Taneike et al., 1991).

In addition to adrenergic and cholinergic nerves, the mammalian reproductive tract is also supplied by peptidergic nerves containing a variety of neuropeptides. These peptidergic nerves vary in type and density among species (Huang et al., 1984; Heinrich et al., 1986). Various neuropeptides are present or coexist in nerve fibres of the female reproductive tract, and these nerves are thought to be involved in the control of reproductive organs. Neuropeptide Y (NPY), vasoactive intestinal polypeptide (VIP), calcitonin gene-related peptide (CGRP), and substance $\mathrm{P}(\mathrm{SP})$ are thought to play important roles in the control of myometrial contractility (Mukai et al., 1991) and uterine blood flow (Ekesbo et al., 1991). As these nerves are particularly abundant in the lower genital tract, they may also play a role in regulation of uterine activity during pregnancy and parturition (Majewski et al., 1995; Houdeau et al., 1997).

In the present study adrenergic innervation of the uteri of genitally normal mares was investigated using the catecholamine enzymatic markers $\mathrm{TH}$ and $\mathrm{D} \beta \mathrm{H}$. The distribution of nerve fibres immunoreactive for the peptide neurotransmitters NPY, CGRP, VIP and SP was also investigated. 


\section{Materials and Methods}

\section{Animals}

Uterine tissue samples were collected from 18 cyclic mares (aged between 3 and 16 years and weighing $390-520 \mathrm{~kg}$ ) killed for reasons other than reproductive problems. The stage of the ovarian cycle was established by determining peripheral blood progesterone concentrations as well as by the presence or absence of a corpus luteum in the ovaries, the presence of a follicle $>30 \mathrm{~mm}$ in diameter and uterine oedema. The reproductive tracts of these mares (seven oestrous mares and 11 dioestrous mares) were removed within 20 min of death and were gently washed and dissected in 0.1 mol PBS $\mathrm{I}^{-1}(\mathrm{pH} 7.2)$. Samples were collected from the uterine horn $(n=4)$, body $(n=4)$ and cervix $(n=4)$ from each mare. Samples were then fixed immediately in $4 \%(\mathrm{w} / \mathrm{v})$ paraformaldehyde $(\mathrm{pH} 7.2)$ for 14-16 h at $4^{\circ} \mathrm{C}$. Samples were transferred into $0.1 \mathrm{~mol}$ PBS $\mathrm{I}^{-1}(\mathrm{pH} 7.2)$ containing $30 \%(\mathrm{v} / \mathrm{v})$ sucrose and stored in this solution for at least $24 \mathrm{~h}$ at $4^{\circ} \mathrm{C}$, with four changes of buffer. Tissues were snap frozen in OCT compound (Miles Inc., Elkhart, IN) in an isopentane-dry-ice slurry and then stored at $-70^{\circ} \mathrm{C}$ before sectioning.

\section{Immunohistochemical procedure}

Cryostat sections 12-15 $\mu \mathrm{m}$ in thickness were cut and mounted on slides coated with Bio-Bond (British BioCell International, Cardiff). Sections were air-dried for 60-90 $\mathrm{min}$ at room temperature, rinsed in $0.01 \mathrm{~mol}$ PBS $\mathrm{I}^{-1}$ $(\mathrm{pH} 7.2)$ for $10 \mathrm{~min}$ and blocked with $3 \% \mathrm{H}_{2} \mathrm{O}_{2}$ in methanol for $30 \mathrm{~min}$ to reduce non-specific binding. Slides were then preincubated with $1 \%$ goat antiserum in 0.01 mol PBS I-1 for $1 \mathrm{~h}$. The excess serum was blotted and the sections were incubated with primary antibody at the appropriate dilution overnight at $4^{\circ} \mathrm{C}$ before washing three times in $0.01 \mathrm{~mol}$ PBS $\left.\right|^{-1}$. Primary antisera were raised in rabbits, and were used at the following dilutions in PBS: TH, 1:6000 (Affiniti Research Products, Exeter); D $\beta H, 1: 4000$ (Affiniti Research Products); NPY, 1:4000 (Affiniti Research Products); VIP, 1:4000 (Peninsula Laboratories Europe, St Helens); CGRP, 1:4000 (Peninsula Laboratories Europe); and SP, 1:6000 (Peninsula Laboratories Europe). The sections were incubated with the second antibody (goat anti-rabbit) in PBS containing $1 \%$ normal goat serum for $2 \mathrm{~h}$ at room temperature. The sections were washed thoroughly three times in PBS, then incubated with the avidin-biotin complex (Vector Laboratories, Cambridge) for a further 30 min and rinsed in PBS. Chromagens (3-amino-9-ethylcarbazole (AEC), red reaction product; or 3,3'-diaminobenzidine $(\mathrm{DAB})$, brown reaction product; Vector Laboratories) were added as the final substrate, and were incubated at room temperature in the dark for 10-15 min. Each slide was then rinsed carefully with distilled water. Slides were mounted and examined by light microscopy.

The specificity of the primary antisera was tested as follows: (a) sections were incubated with antibody that had been preabsorbed with its synthetic antigen $(10 \mu \mathrm{g}$ of antigen per $\mathrm{ml}$ diluted antiserum) in our laboratory (Corcoran, 1996); (b) the primary antibody was omitted from the incubation; or (c) normal rabbit serum was substituted for the primary antibody. Positive controls comprised equine jejunum and feline uterus incubated with each of the primary antibodies.

The sections were examined histologically under the $\times 40$ objective of a microscope, and localization and distribution of the immunoreactive nerves were classified depending on the density of staining assessed by subjective observation, and graded semiquantitatively as: - , not found; \pm , very few; + , a very small number of fibres; ++ , a small number of fibres; +++ , a moderate number of fibres; ++++ , a large number of fibres; and +++++ , a very large number of fibres.

\section{Results}

All results are based on tissue material from 18 cyclic mares (seven oestrous mares and 11 dioestrous mares) (Table 1). At least two sections were examined from each histological block ( $n=8$ sections per site for each mare). A large number of adrenergic nerves immunoreactive for the catecholaminesynthesizing enzymes $\mathrm{TH}$ and $\mathrm{D} \beta \mathrm{H}$ were present in all regions of the uterus. Adrenergic nerves were present at a greater density than were peptidergic nerves. There was no difference in the density or distribution of innervation between oestrous and dioestrous mares (data not shown) with subjective observation and so sections from all mares were included in one group, irrespective of stage of the cycle.

\section{Distribution of adrenergic nerves ( $T H$ and $D \beta H$ )}

Nerves immunoreactive (IR) for $\mathrm{TH}$ and $\mathrm{D} \beta \mathrm{H}$ were found throughout all regions of the uterus. Numerous TH-IR nerve bundles and fibres (Fig. 1a) were found in the uterine horn, body and the cervix. In the muscle layers, nerve fibres containing TH-IR often travelled parallel to the muscle fibres. Nerve fibres immunoreactive for $\mathrm{TH}$ were distributed along the vessels (Fig. 1b) extending from the longitudinal to the circular smooth muscle layers of the myometrium. The density of immunoreactivity for $\mathrm{TH}$ was greater in the myometrium than in the endometrium. Some nerve fibres entered the endometrium close to endometrial glands (Fig. 1c). Nerves immunoreactive for $\mathrm{D} \beta \mathrm{H}$ were also present in all regions of the uterus. An intense network of nerve bundles and fibres containing D $\beta H$-IR was found in the uterine horn (Fig. 2a), body and the cervix (Fig. 2b). Nerves containing $\mathrm{D} \beta \mathrm{H}$ were present in both myometrium (Fig. 2c) and endometrium, and were often located close to blood vessels (Fig. 2b). The density and distribution for $\mathrm{D} \beta \mathrm{H}$ was similar to that for $\mathrm{TH}$.

\section{Distribution of peptidergic nerves (NPY, VIP, CGRP and $S P)$}

Moderate numbers of nerve fibres containing NPY-IR were found in all regions of the uterus. Nerve fibres 
Table 1. Distribution and relative density of adrenergic- and peptidergic-immunoreactive nerves in the equine uterine horn, body and cervix

\begin{tabular}{|c|c|c|c|c|c|c|}
\hline \multirow[b]{2}{*}{ Regions } & \multicolumn{2}{|c|}{ Catecholamine synthesizing enzymes } & \multicolumn{4}{|c|}{ Neuropeptides } \\
\hline & $\mathrm{TH}$ & $\mathrm{D} \beta \mathrm{H}$ & NPY & VIP & CGRP & SP \\
\hline \multicolumn{7}{|l|}{ Uterine horn } \\
\hline Endometrium & +++ & +++ & ++ & + & + & - \\
\hline Myometrium & +++++ & +++++ & +++ & + & + & - \\
\hline Vascular layer & +++++ & +++++ & ++++ & \pm & \pm & - \\
\hline \multicolumn{7}{|l|}{ Uterine body } \\
\hline Endometrium & +++ & +++ & ++ & + & + & - \\
\hline Myometrium & +++++ & +++++ & +++ & + & + & - \\
\hline Vascular layer & +++++ & +++++ & ++++ & \pm & \pm & - \\
\hline \multicolumn{7}{|l|}{ Cervix } \\
\hline Endocervix & +++ & +++ & ++ & + & + & - \\
\hline Myocervix & +++++ & +++++ & ++++ & ++ & ++ & \pm \\
\hline Vascular layer & +++++ & +++++ & ++++ & \pm & \pm & - \\
\hline
\end{tabular}

Eight sections were examined at each site for each mare.

CGRP: calcitonin gene-related peptide; D $\beta \mathrm{H}$ : dopamine $\beta$-hydroxylase; NPY: neuropeptide Y; SP: substance P; TH: tyrosine hydroxylase; VIP: vasoactive intestinal polypeptide.

Semiquantitative evaluation of the density of the adrenergic- and peptidergic-immunoreactive nerve fibres graded as: - , not found; \pm , very few; + , a very small number of fibres; ++, a small number of fibres; +++, a moderate number of fibres; ++++, a large number of fibres; and +++++, a very large number of fibres.

containing NPY were present in both the circular and longitudinal muscle layers travelling in parallel to the long axis of the smooth muscle layers (Fig. 3a) and often associated with blood vessels forming vessel-surrounding plexiform networks (Fig. 3b). The density of NPY was greater in the myometrium than in the endometrium (Table 1). Nerve fibres containing NPY-IR were seen in the endometrium beneath the luminal epithelium, near endometrial glands (Fig. 3c) and submucosa. Nerve cell bodies immunoreactive for NPY were also observed.

The distribution of nerve fibres containing VIP-IR was similar to that of nerve fibres containing NPY-IR but there were fewer of them. A small number of scattered nerve fibres containing VIP-IR were seen in the interstitial connective tissue and along the smooth muscle layers (Fig. 4a) occasionally associated with blood vessels. In the endometrium, nerve fibres containing VIP-IR were seen around the endometrial glands (Fig. 4b). The numbers of nerves containing VIP-IR were similar in the uterine horn and body, but were higher in the cervix (Table 1).

Nerve fibres containing CGRP-IR were less numerous than nerves containing NPY, but were present in similar density to nerve fibres containing VIP. A small number of scattered nerve fibres containing CGRP were seen in the interstitial connective tissue and along smooth muscle layers (Fig. 5a) occasionally associated with blood vessels. Thick bundles of nerve fibres containing CGRP-IR were found in the cervix, travelling parallel to smooth muscle bundles (Fig. 5b). There were more nerve fibres containing CGRP-IR in the cervix than in other parts of uterus.

A few nerve fibres containing SP-IR were found only in the cervix. Nerve fibres containing SP-IR travelled parallel to the long axis of the smooth muscle bundles, close to blood vessels (Fig. 6).

Negative control sections (omission or preabsorption of primary antibody) failed to demonstrate any positive staining (Fig. 7) and positive controls showed strong immunoreactivity.

\section{Discussion}

This report is the first description of the distribution of adrenergic and peptidergic nerves in different regions of equine uterus. The present results provide immunohistochemical evidence for an abundant adrenergic innervation, as well as the presence of peptidergic innervation containing the neuropeptides, NPY, VIP, CGRP and SP, in the uterus of mares.

Previous studies have reported that the mammalian female reproductive tract receives several types of nerve fibres (Renegar and Rexroad, 1990; Melo and Machado, 1993), including the classical autonomic nervous system (sympathetic and parasympathetic), and peptidergic nerves containing various neuropeptides. Although the relative densities of different classes of nerve fibre vary among different reproductive organs and among species, their distribution patterns tend to be similar. In most species, the reproductive tract has rich adrenergic innervation associated with blood vessels. These nerves are composed of long postganglionic nerve fibres, the cell bodies of which are in the lumbar and mesenteric ganglia and short postganglionic nerve fibres originating in the pelvic ganglia (Sjöberg, 1967). These 'short' adrenergic nerves are believed to innervate predominantly the smooth muscle of the reproductive 

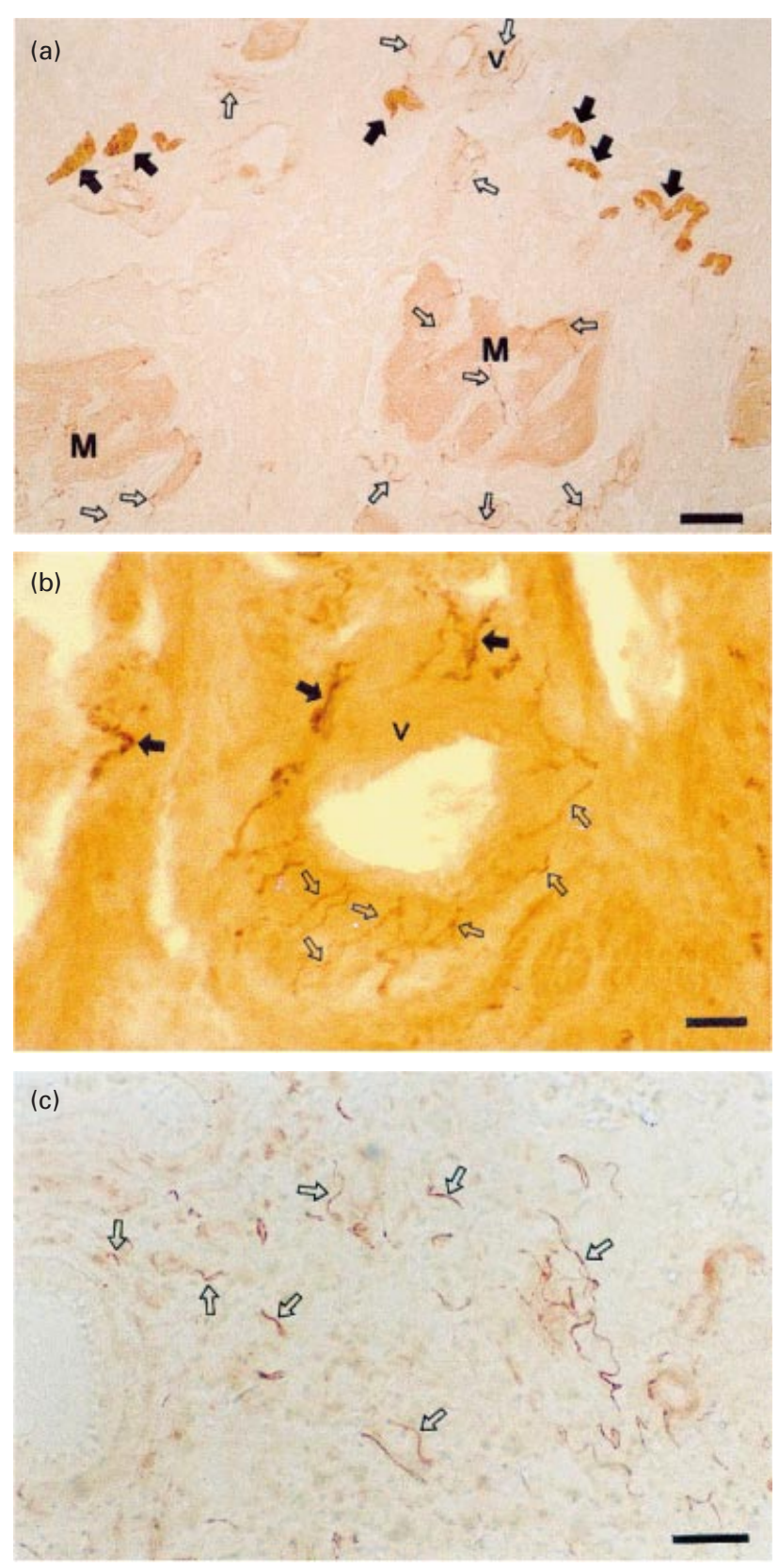

Fig. 1. Tyrosine hydroxylase (TH)-immunoreactive (IR) nerve fibres in different uterine regions in the mare. (a) TH-IR nerve bundles (solid arrows) and nerve fibres (open arrows) in the myometrium of the uterine body. M: smooth muscle bundles; V: blood vessel. (b) TH-IR nerve bundles (solid arrows) and nerve fibres (open arrows) in the myometrium of the uterine horn associated with blood vessels (V). (c) TH-IR nerve fibres (open arrows) close to the endometrial glands in the endometrium of the uterine body. Scale bars represent $250 \mu \mathrm{m}$ (a) and $50 \mu \mathrm{m}$ (b,c).

organs and they differ from the long nerve fibres anatomically. The most interesting feature of these short adrenergic nerves is the regulation of transmitter (noradrenaline) metabolism in these nerve fibres by ovarian steroid hormones (Owman and Sjöberg, 1973; Marshall, 1981). In cyclic animals, the
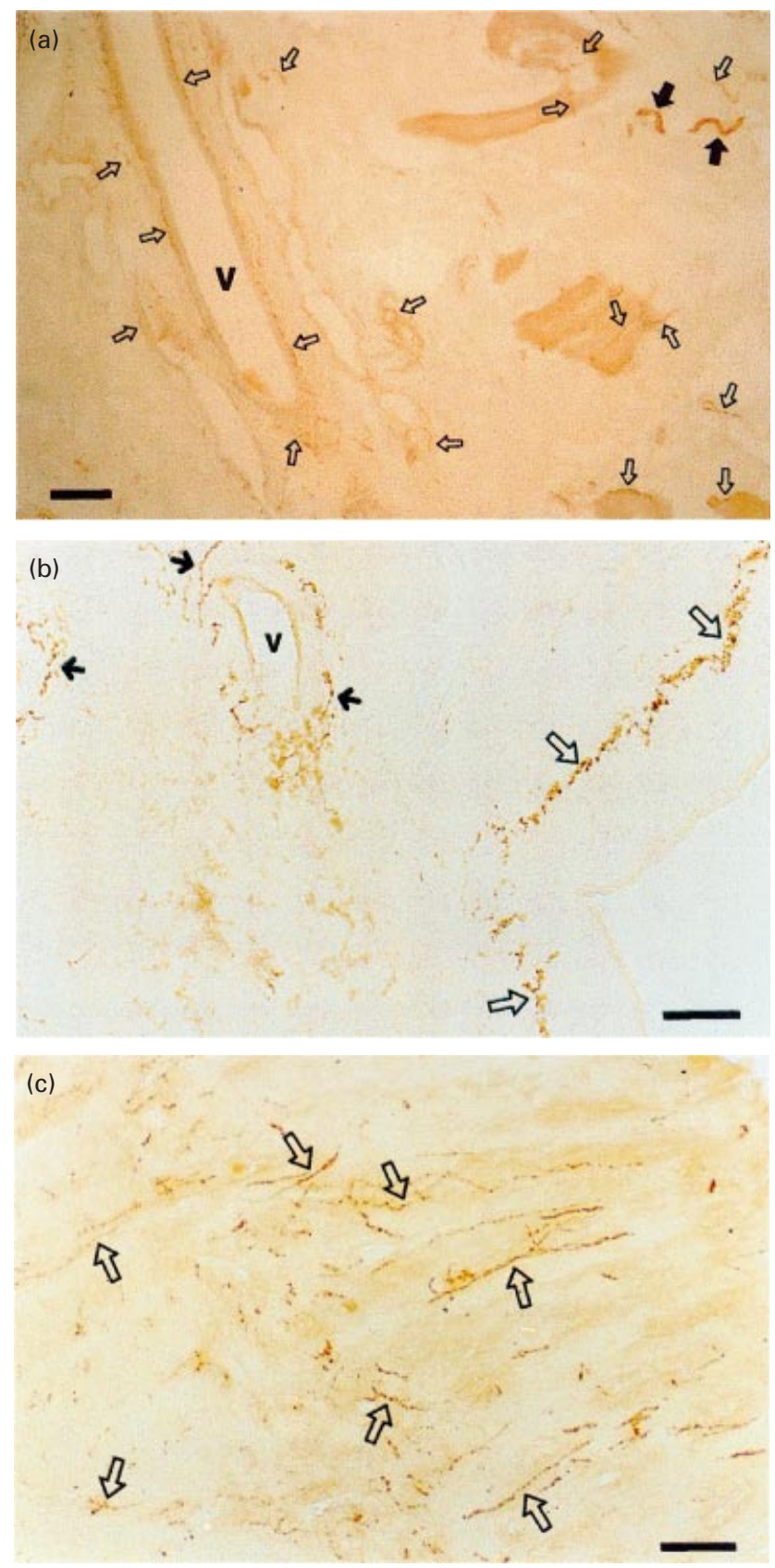

Fig. 2. Dopamine $\beta$-hydroxylase (DßH)-immunoreactive (IR) nerve fibres in different uterine regions in the mare. (a) D $\mathrm{DH}$-IR nerve bundles (solid arrows) and nerve fibres (open arrows) in the myometrium of the uterine horn. V: blood vessel. (b) D $\beta H$-IR nerve fibres (open arrows) in the cervix and nerve fibres (solid arrows) associated with blood vessels $(\mathrm{V})$. (c) D $\beta \mathrm{H}$-IR nerve fibres (nerve bundles; open arrows) in the smooth muscle layers of the uterine body. Scale bars represent $250 \mu \mathrm{m}$ (a), $50 \mu \mathrm{m}$ (b) and $100 \mu \mathrm{m}$ (c).

changes in uterine innervation vary among species. Melo and Machado (1993) and Wrobel and Kujat (1993) reported that the density and distribution of the nerve fibres was nearly the same during oestrus as in dioestrus in cows, whereas in rats, Zoubina et al. (1998) reported that a 

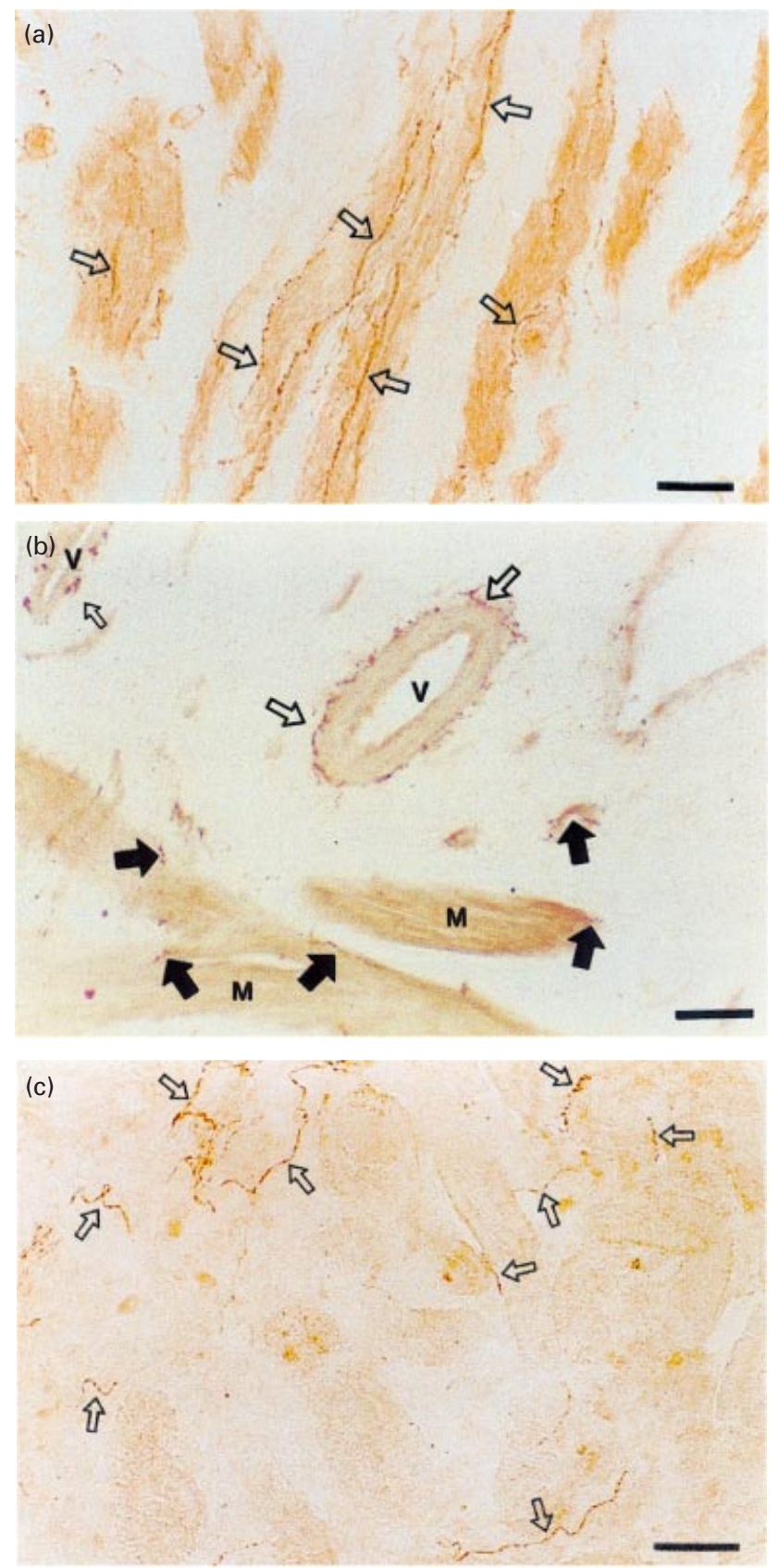

Fig. 3. Neuropeptide $Y(N P Y)$-immunoreactive (IR) nerve fibres in different uterine regions in the mare. (a) NPY-IR nerve fibres (open arrows) in the outer longitudinal smooth muscle layer of the uterine horn. (b) NPY-IR nerve fibres (solid arrows) in the smooth muscle bundles $(\mathrm{M})$ and nerve fibres (open arrows) associated with blood vessels (V) in the cervix. (c) NPY-IR nerve fibres (open arrows) close to the endometrial glands of the uterine body. Scale bars represent $50 \mu \mathrm{m}$.

reduction in the numbers of nerves occurred during oestrus and was followed by subsequent restoration of innervation. In the present study in mares, stage of cycle did not appear to influence uterine or cervical innervation.

In the present study, $\mathrm{TH}$ - and $\mathrm{D} \beta \mathrm{H}-\mathrm{IR}$ nerves had a (a)

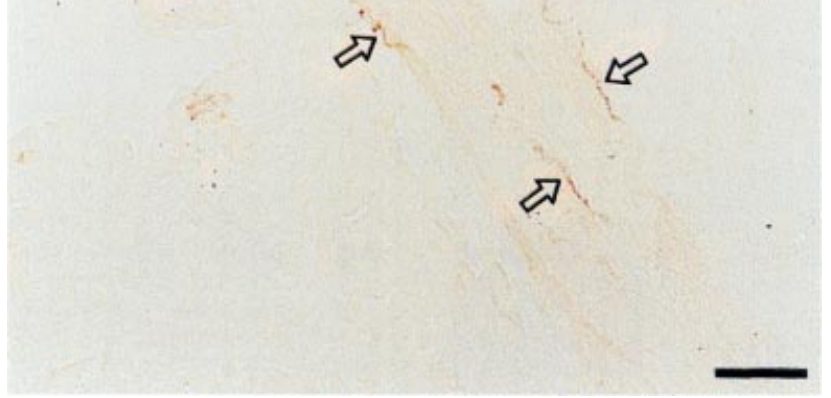

(b)

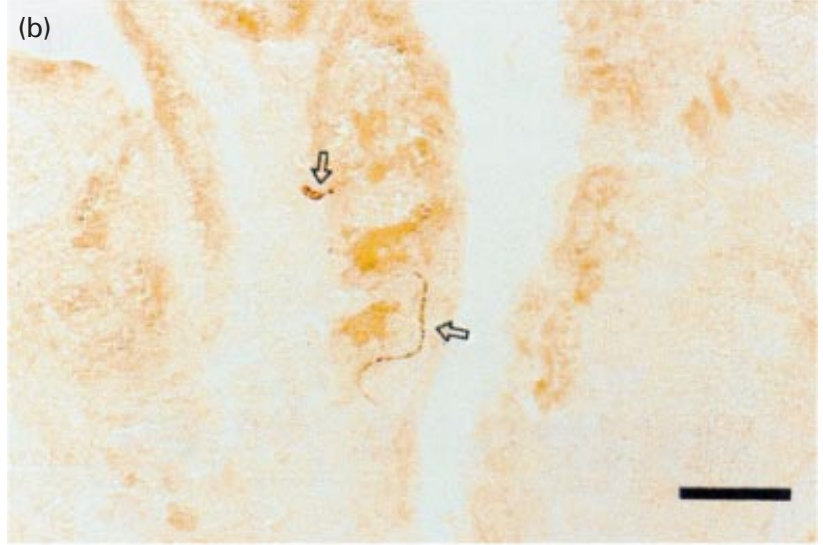

Fig. 4. Vasoactive intestinal polypeptide (VIP)-immunoreactive (IR) nerve fibres in different uterine regions in the mare. (a) VIP-IR nerve fibres (open arrows) in the myometrium of the uterine body. (b) VIP-IR nerve fibres (open arrows) in the endometrium of the uterine horn. Scale bars represent $50 \mu \mathrm{m}$.

similar distribution and both enzymatic markers were probably demonstrating the presence of noradrenaline. As in other species, intense adrenergic innervation was present in the smooth muscle layers often associated with blood vessels, and in the endometrium. In the myometrium, abundant thick nerve bundles were located with smooth muscle bundles and, in the endometrium, adrenergic nerve fibres were found in endometrial glands and near endometrial vessels, but nerve bundles were seen only rarely. These adrenergic nerve fibres associated with blood vessels, endometrial glands and myometrial smooth muscle probably function in the regulation of uterine blood flow, endometrial secretion and uterine contractility (Marshall, 1981; Renegar and Rexroad, 1990).

The distribution of the peptidergic nerves in the equine uterus was similar to that reported in guinea-pigs (Alm and Lundberg, 1988; Mitchell and Ahmed, 1992), rats (Serghini et al., 1998), sows (Taneike et al., 1991; Majewski et al., 1995) and women (Blank et al., 1986; Jørgensen, 1994). Of the neuropeptides, NPY-IR nerve fibres were particularly numerous in the equine uterus. NPY-IR nerves appeared to have fewer nerve fibre bundles than did the adrenergic 


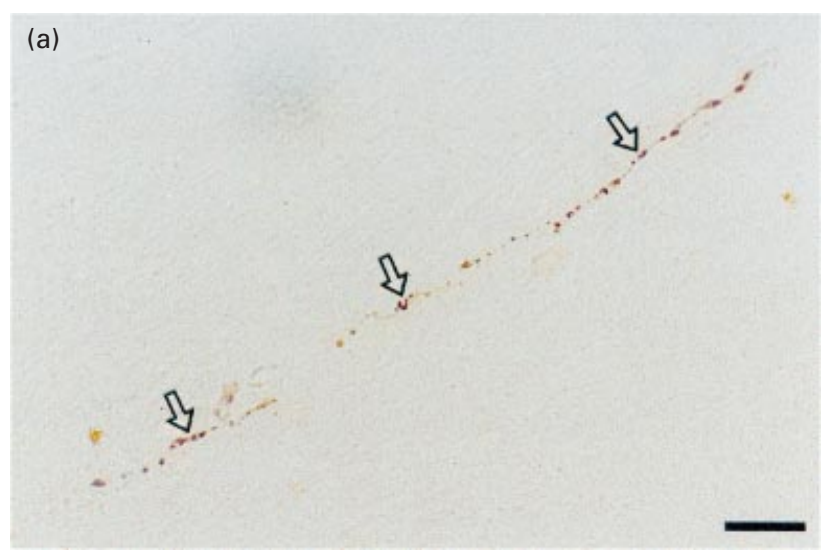

(b)

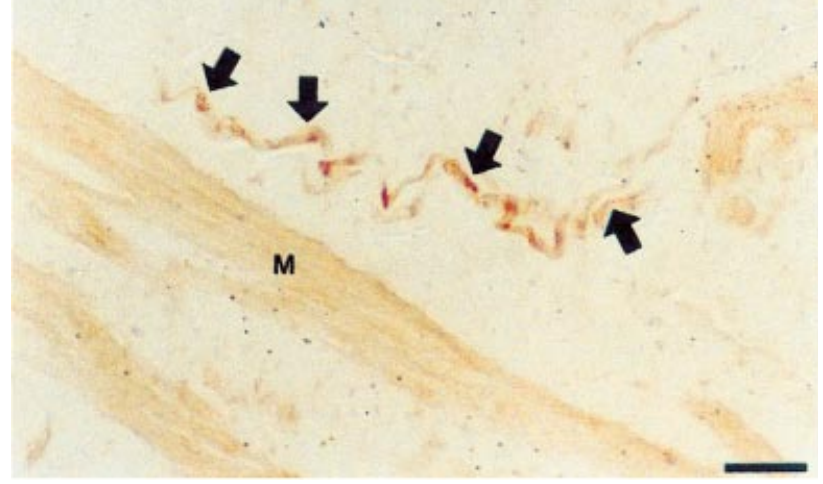

Fig. 5. Calcitonin gene-related peptide (CGRP)-immunoreactive (IR) nerve fibres in different uterine regions in the mare. (a) CGRPIR nerve fibres (open arrows) in the myometrium of the uterine body. (b) Thin CGRP-IR nerve bundles and fibres (solid arrows) in the cervix. M: smooth muscle bundles. Scale bars represent $50 \mu \mathrm{m}$.

nerves, but their pattern of distribution was similar to that of adrenergic innervation. These findings indicate that most NPY-IR nerve fibres in the equine genital tract co-exist with $\mathrm{TH}-/ \mathrm{D} \beta \mathrm{H}-\mathrm{IR}$ nerves as documented in other species (Alm and Lundberg, 1988; Mitchell and Ahmed, 1992), but this needs to be confirmed by double-immunostaining. Previous quantitative studies have shown that NPY concentrations are greater in the cervix than in the uterine body of rats (Serghini et al., 1998) and women (Blank et al., 1986; Jørgensen, 1994). However, in guinea-pigs, concentrations were greater in the uterine body (Huang et al., 1984). In contrast, an immunohistochemical study showed that abundant NPYIR nerves were present in all regions in the guinea-pig genital tract (Alm and Lundberg, 1988). In the present study in mares, the density and distribution of NPY-IR nerve fibres were similar in the uterine horn, body and the cervix.

A small number of fine nerve fibres containing VIP-IR and CGRP-IR were found in the equine uterus and the density of these nerve fibres was greater in the cervical region. In the myometrium, nerve fibres containing VIP and CGRP were found in the smooth muscle layers often associated with blood vessels, and nerve fibre bundles were

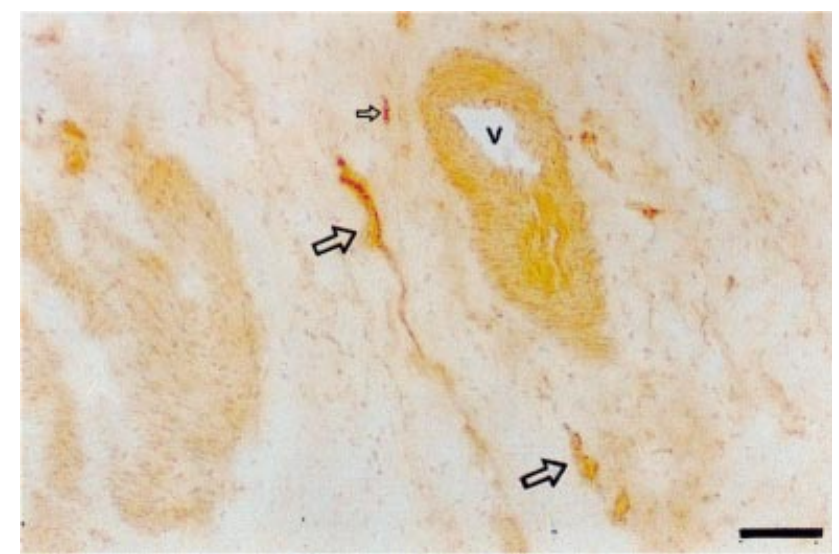

Fig. 6. Substance $P$ (SP)-immunoreactive nerve fibres (open arrows) near blood vessel (V) in inner smooth muscle layer in the equine cervix. Scale bar represents $50 \mu \mathrm{m}$.

found only rarely. In the endometrium, nerve fibres containing VIP and CGRP were found near endometrial glands. These findings are in agreement with reports in other species (Heinrich et al., 1986; Ottensen and Fahrenkrug, 1995), although there are differences in the density of distribution of the neuropeptides reported among species. In cats (Alm et al., 1986), mice (Huang et al., 1984) and women (Blank et al., 1986), nerve fibres containing VIP-IR are abundant in the uterus, whereas guinea-pigs have only a small number (Heinrich et al., 1986; Alm and Lundberg, 1988). In contrast, the density of CGRP is similar in all species. The small number of nerve fibres containing CGRP found in the female reproductive tract has been reported to be slightly denser in the cervix than in the uterine horn and body in sows (Lakomy et al., 1994).

Substance $P$ is the least frequently reported neuropeptide transmitter in the female reproductive tract (Huang et al., 1984; Majewski et al., 1995) and was found only rarely in the uteri of women or guinea-pigs (Heinrich et al., 1986; Mitchell and Ahmed, 1992). In the present study in mares, SP-immunoreactive fibres were present in the cervix only.

Many roles have been suggested for these neuropeptides in the female reproductive tract. The density of the peptidergic nerves is less than that of adrenergic nerves, but it is clear that neuropeptides have a role in controlling female reproductive function. NPY is known to coexist with noradrenaline in most sympathetic vasoconstrictor nerve fibres (Lundberg et al., 1990; Morris, 1990) and seems to have important biological functions in the regulation of vascular tone and myometrial contractility (Stjernquist and Owman, 1987; Atke et al., 1996). The presence of nerves containing VIP in the female reproductive tract, as well as the dose-dependent inhibitory effect of VIP on its smooth muscle activity, indicates that VIP plays a role in the local control of smooth muscle (Helm et al., 1981; Ottensen et al., 1983). VIP is a potent dilator of the uterine artery (Morris and Murphy, 1988). SP is known to produce an increase in 


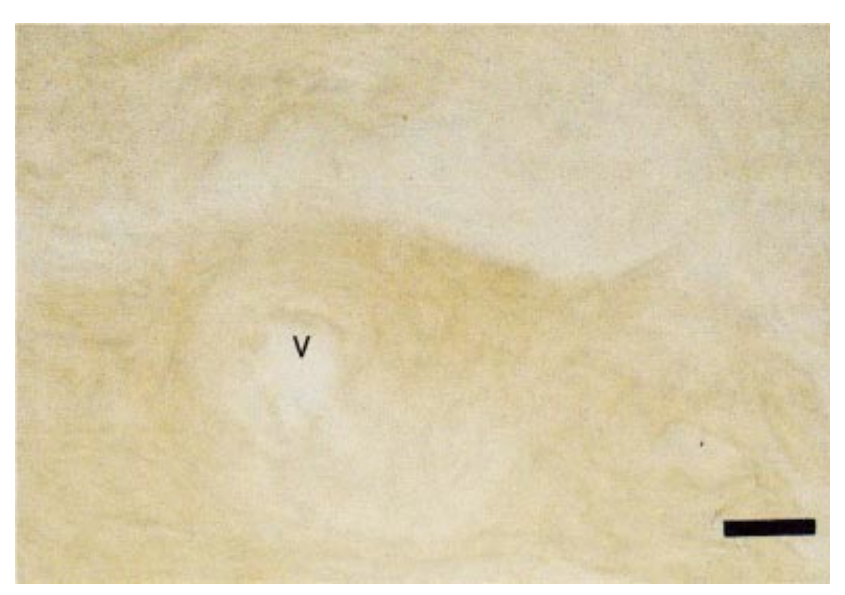

Fig. 7. Negative control of equine uterine horn. V: blood vessel. Scale bar represents $50 \mu \mathrm{m}$.

myometrial blood flow and to stimulate both mechanical and myoelectrical activity (Ottensen et al., 1983). CGRP and SP are present and coexist in some uterine afferent nerves and influence uterine contractility: SP has a contractile effect and CGRP has a relaxing effect (Shew et al., 1991). The present results showed peptidergic nerve fibres coursing beneath the luminal epithelium and endometrial glands in the endometrium, in agreement with previous observations (Mitchell and Ahmed, 1992) and indicating a role for neurogenic mechanisms in the regulation of mucous secretion and endometrial secretion in the equine reproductive tract, as in other species (Blank et al., 1986; Jørgensen, 1994; Atke et al., 1996).

The presence and location of adrenergic and peptidergic innervation in the equine uterus and cervix indicate an important role for these neuronal factors in the regulation of uterine motility, endometrial secretion and blood flow. The fact that the adrenergic and peptidergic nerves are located predominantly in the myometrium indicates that neurogenic factors have an important role in controlling uterine contractility. The high density of innervation in the cervix indicates a role in regulating cervical closure in dioestrus and during pregnancy, and in relaxation of the cervix at oestrus and during parturition in mares. However, the exact functions of specific nerve fibres in the female reproductive tract remain unclear. Further studies are required to clarify their precise physiological role in the regulatory mechanisms of the equine reproductive tract.

The authors wish to thank Nobuo Kitamura (Obihiro University, Japan) for technical advice. They also thank the Department of Veterinary Clinical Studies, Royal (Dick) School of Veterinary Medicine, University of Edinburgh for financial support.

\section{References}

Alm P, Owman C, Sjöberg N, Stiernquist M and Sundler F (1986) Histochemical demonstration of a concomitant reduction in neural vasoactive intestinal polypeptide, acetylcholinesterase, and noradrenaline of cat uterus during pregnancy Neuroscience 18 713-726

Alm P and Lundberg L-M (1988) Co-existence and origin of peptidergic and adrenergic nerves in the guinea pig uterus. Retrograde tracing and immunocytochemistry, effects of chemical sympathectomy, capsalcin treatment and pregnancy Cell and Tissue Research 254 517-530

Atke A, Henriksen JS, Jacobsen HS and Vilhardt H (1996) Characterization of the rat myometrial contractility response to neuropeptide $\mathrm{Y}$ Journal of Receptor and Signal Transduction Research 16 25-38

Blank MA, Gu J, Allen JM et al. (1986) The regional distribution of NPY-, PHM-, and VIP-containing nerves in the human female genital tract International Journal of Fertility 31 218-222

Corcoran BM (1996) Distribution of calcitonin gene-related peptide, vasoactive intestinal polypeptide, neuropeptide $Y$, substance $P$ and dopamine $B$-hydroxylase immunoreactive nerve fibres in the trachea of sheep Research in Veterinary Science 60 69-75

Ekesbo R, Alm P, Lundberg LM and Akerlund M (1991) Innervation of the human uterine artery and contractile responses to neuropeptides Gynecologic and Obstetric Investigation 31 30-36

Heinrich D, Reinecke M and Forssmann WG (1986) Peptidergic innervation of the human and guinea pig uterus Archives of Gynecology $237213-219$

Helm G, Ottesen B, Fahrenkrug J, Larsen JJ, Owman C, Sjöberg NO, Stölberg B, Sundler F and Walles B (1981) Vasoactive intestinal polypeptide (VIP) in the human female reproductive tract: distribution and motor effects Biology of Reproduction 25 227-234

Houdeau E, Boyer P-A, Rousseau A and Rousseau J-P (1997) Coexpression of neuropeptide $\mathrm{Y}$ and vasoactive intestinal polypeptide in pelvic plexus neurones innervating the uterus and cervix in the rat Cell and Tissue Research 288 285-292

Huang WM, Gu J, Blank MA, Allen JM, Bloom SR and Polak JM (1984) Peptide-immunoreactive nerves in the mammalian female genital tract Histochemical Journal 16 1297-1310

Jørgensen JC (1994) Neuropeptide $Y$ in mammalian genital tract: localization and biological action Danish Medical Bulletin 41 294-305

Lakomy M, Häppölä O, Majewski M and Kaleczyc J (1994) Immunohistochemical localization of neuropeptides in nerve fibres of the porcine vagina and uterine cervix Follia Histochemica et Cytobiologica 32 167-175

Lundberg JM, Franco-Cereceda A, Hemsen A, Lacroix JS and Pernow J (1990) Pharmacology of noradrenaline and neuropeptide tyrosine (NPY)-mediated sympathetic cotransmission Fundamental Clinical Pharmacology 4 373-391

Majewski M, Sienkiewicz W, Kaleczyc J, Mayer B, Czaja K and Lakomy M (1995) The distribution and co-localization of immunoreactivity to nitric oxide synthase, vasoactive intestinal polypeptide and substance $\mathrm{P}$ within nerve fibres supplying bovine and porcine female genital organs Cell and Tissue Research 281 445-464

Marshall JM (1981) Effects of ovarian steroids and pregnancy on adrenergic nerves of uterus and oviduct American Journal of Physiology $\mathbf{2 4 0}$ C165-C174

Melo RCN and Machado CRS (1993) Noradrenergic and acetycholineesterase-positive nerve fibres of the uterus in sexually immature and cycling rats Histochemical Journal 25 213-218

Mitchell RS and Ahmed E (1992) An immunohistochemical study of the catecholamine synthesizing enzymes and neuropeptides in the female guinea-pig uterus and vagina Histochemical Journal 24 361-367

Morris JL and Murphy R (1988) Evidence that neuropeptide Y released from noradrenergic axons causes prolonged contraction of the guinea-pig uterine artery Journal of Autonomic Nervous System 24 241-249

Mukai H, Tanaka H, Goto K, Kawai K, Yamashita K and Munekata E (1991) Structure-activity relationships of mammalian bombesin-like neuropeptides in the contraction of rat uterus Neuropeptides 19 243-250

Nikolakopoulos E and Watson ED (1997) Uterine motility in mares resistant and susceptible to endometritis Journal of Reproduction and Fertility Abstract Series 20 Abstract 32

Ottesen B and Fahrenkrug J (1995) Vasoactive intestinal polypeptide and other preprovasoactive intestinal polypeptide-derived peptides in the female and male genital tract: localization, biosynthesis, and clinical 
significance American Journal of Obstetrics and Gynecology 172 $1615-1631$

Ottensen B, Gram BR and Fahrenkrug J (1983) Neuropeptides in the female genital tract: effect on vascular and non-vascular smooth muscle Peptides 4 387-392

Owman C and Sjöberg NO (1973) Effect of pregnancy and sex hormones on the transmitter level in uterine short adrenergic neurons. In Frontiers in Catecholamine Research pp 795-801 Eds E Usdin and SH Snyder. Pergamon, New York

Renegar RH and Rexroad CE, Jr (1990) Uterine adrenergic and cholinesterase-positive nerves and myometrial catecholamine concentrations during pregnancy in sheep Acta Anatomica 137 373-381

Serghini R, Prud'homme MJ, Vaudry H and Roussea JP (1998) The suprarenal ganglia: origin of the neuropeptide $\mathrm{Y}$ innervation of the upper part of the uterus in the rat Annals New York Academy of Sciences 839 621-624

Shew RL, Papka RE and McNeill DL (1991) Substance P and calcitonin gene-related peptide immunoreactivity in nerves of the rat uterus: localization, colocalization and effects on uterine contractility Peptides 12 593-600

Sjöberg NO (1967) The adrenergic transmitter of the female reproductive tract: distribution and functional changes Acta Physiologica Scandinavica Supplementum 305 5-26

Stjernquist M and Owman C (1987) Interaction of noradrenaline, NPY and
VIP with the neurogenic cholinergic response of the rat uterine cervix in vitro. Acta Physiologica Scandinavica 131 553-562

Taneike T, Miyazaki H, Nakamura H and Ohga A (1991) Autonomic innervation of the circular and longitudinal layers in swine myometrium Biology of Reproduction 45 831-840

Thorbert G, Alm P, Owman C and Sjöberg NO (1977) Regional distribution of autonomic nerves in guinea-pig uterus American Journal of Physiology 233 C25-C34

Troedsson MHT, Liu IKM, Ing M, Pascoe J and Thurmond M (1993) Multiple site electromyography recordings of uterine activity following an intrauterine bacterial challenge in mares susceptible and resistant to chronic uterine infection Journal of Reproduction and Fertility 99 307-313

Wrobel K-H and Kujat R (1993) The bovine tubouterine junction: general innervation pattern and distribution of adrenergic, cholinergic, and peptidergic nerve fibres Cell and Tissue Research 274 493-501

Zoubina EV, Fan Q and Smith PG (1998) Variation in uterine innervation during the estrous cycle in rat Journal of Comparative Neurology 397 $561-571$

Received 22 December 2000.

First decision 19 February 2001.

Accepted 2 April 2001. 